\title{
Improving a Case-Control Study of Multiple Sclerosis Using Formative Research
}

\author{
Dhelia M. Williamson, Ph.D ${ }^{1, *}$, Laurie Wagner, M.P.H. ${ }^{2}$; Judy P. Henry, Ph.D ${ }^{3}$ \\ ${ }^{1}$ Division of Reproductive Health, Centers for Disease Control and Prevention, Atlanta, Georgia, 30333, United States \\ ${ }^{2}$ McKing Consulting Corporation, Atlanta, Georgia, 30341 \\ ${ }^{3}$ Austin/Travis County Health and Human Services, Austin, Texas, 78702, United States \\ *Corresponding author: djw8@cdc.gov
}

Copyright $(2013$ Horizon Research Publishing All rights reserved.

\begin{abstract}
Formative research (i.e. focus groups and key informant interviews) was conducted to understand risk perceptions and identify barriers to participation in a case-control study of environmental exposures and genetic susceptibility as risk factors for multiple sclerosis (MS). Individuals with MS were recruited to participate in a focus group discussion and individual interviews. Participants were asked to review and comment on study materials and process including participation, interview, genetic testing, confidentiality, and questionnaire. A structured discussion guide was used with all participants to ensure uniformity and coverage of all predetermined topics. Participants reported an increased likelihood of participation if they were informed about the study by their neurologist and not a government agency. All participants expressed willingness to provide a blood sample for genotyping but disagreed about the setting for the blood draw (at home or in a lab). Participants were concerned that they would not receive their individual genotyping results. The study protocol and materials were revised based on comments from the focus group participants. Formative research is an under-utilized resource for researchers conducting epidemiologic studies. Even with limited resources, piloting study materials with individuals similar to the proposed study population can provide opportunities to make modifications to effectively meet the needs of participants and promote participation and retention.
\end{abstract}

Keywords Case-Control Study, Environmental Risk Factors, Formative Research, Genetic Susceptibility, Multiple Sclerosis

\section{Introduction}

Recent advances in DNA technology have allowed new discoveries about the genetic basis of many diseases, including genetic susceptibility to common chronic diseases (1). The etiologic basis of most common diseases, however, is multifactorial, so even though a genetic mutation may increase risk, the disease is also influenced by environmental exposures, behaviors and other genes, and interaction between some or all etiologic factors (2). Epidemiologic studies play a key role in identifying these associations; however, achieving high participation rates in these studies has become increasingly more difficult (3). Before conducting such a study, researchers attempt to identify and address conditions that may pose particular challenges for participants that would decrease their likelihood of participation (3). For instance, it has been shown that contacting participants multiple times and providing choices improves recruitment (4).

The development of creative recruitment and retention techniques that optimize participation need to be customized to the targeted population (3). This is especially true in studies that collect a biologic specimen since the collection of specimens can be a barrier to participation $(5,6)$. Therefore, it is essential that potential barriers to participation are identified and addressed before a study is conducted.

Formative research (i.e. research conducted to examine behaviors, attitudes and practices of a target group, including exploring behavioral determinants) has been used to design effective public health activities using both qualitative and quantitative methods (6). These types of activities would be beneficial during the planning phase of epidemiologic studies to identify concerns, enhance participant recruitment, identify potential obstacles and test or validate questionnaires; however, they are not routinely used (7 - 10). Before conducting a case-control study of multiple sclerosis (MS) that included collecting a blood sample for genotyping, the Agency for Toxic Substances and Disease Registry (ATSDR), a federal public health agency, and the Texas Department of State Health Services (TDSHS) wanted to gain an understanding of risk perceptions potential participants may have that could impede study participation. Individuals with MS were identified and asked to participate in a focus group discussion or individual interviews, to learn about perceptions, attitudes, and beliefs related to the 
case-control study goals, recruitment methods, questionnaire topics, specimen collection, and dissemination of results.

Formative research was conducted to evaluate a proposed case-control study of MS. The purpose of this paper is to describe the focus group and interview process, including identification and selection of participants, group and telephone interview procedures, and modification of study materials. Results from the actual study that was conducted are provided to illustrate how the input from the focus group improved the study. We also provide lessons learned by the investigators and recommendations for other researchers who conduct community studies that include a genetic component.

\section{Methods}

\subsection{Proposed Case-Control Study}

MS is a complex disease with a multi-factorial etiology, although the specific biological mechanism of the disease remains unknown. In the proposed study, participants would be asked to complete a questionnaire and provide a blood sample for genotyping. The questionnaire was designed to collect information pertaining to exposure to environmental factors thought to be associated with MS such as heavy metals, solvents and other toxic chemicals. It included a complete residential history, location of schools attended, occupational history and hobbies/lifestyle exposures. Information would also be collected regarding family history, medical history, reproductive history, and smoking. Cases would complete one extra section regarding the course of their disease. A blood sample would be obtained from all participants (cases and controls) to genotype single nucleotide polymorphisms (SNPs) in genes associated with MS. Genotyping results would be reported in summary form but not given back to individuals.

\subsection{Focus Group Participants}

Participants were individuals with MS who were identified in a cluster investigation in El Paso, Texas examining childhood exposure to heavy metals from a smelter and the risk of developing MS (11). At the end of the study, individuals identified with MS were asked if they would be willing to participate in future MS studies. Those individuals that agreed were contacted by TDSHS and asked to participate in a focus group.

\subsection{Human Subjects}

This project was determined to be exempt from human subjects regulations at 45 CFR 46.101 (b)(2a) because interviews with adults were conducted which did not result in any identifiable data. Focus group participants provided verbal consent to participate and did not receive any compensation.

\subsection{Setting}

Two different sessions were held to accommodate the individuals who agreed to participate. First, an in-person focus group meeting was conducted at the TDSHS in Austin, Texas for those individuals who lived locally. Second, telephone discussions were conducted individually with those participants who lived outside the Austin area.

\subsection{Procedures}

For the in-person meeting, a circular sitting arrangement was used to reduce barriers and facilitate discussion and focus group members were introduced using only first names. The facilitator provided a brief overview of how the focus group discussion would proceed and described the background work the health department had conducted regarding multiple sclerosis in Texas. An overview of the proposed study, including the design, recruitment of study participants, data collection, genotyping, and confidentiality was also provided.

Participants were encouraged to provide their opinions and to explore their views with other participants. Participants were encouraged to speak freely and all the facilitators documented responses. The discussion lasted approximately two hours. Telephone discussions were held the following day using the same outline as the in-person focus group and ranged from 30 minutes to one hour.

Due to concerns of confidentiality on the part of the participants neither the focus group nor the telephone discussions were audio-taped. Facilitators took notes during the meeting to record opinions of the participants. Specific recommendations discussed during the meeting were reviewed before participants were dismissed to ensure accurate reporting.

\subsection{Identifying Obstacles to Participation and Risk Perceptions}

A structured discussion guide was used for both the focus group and the telephone interviews to ensure uniformity and coverage of all predetermined topics. All participants were asked for their opinions regarding the issues listed in Table 1.

\subsection{Analytic Approach}

After completion of the focus group and phone interviews, the facilitators discussed their overall impressions of the opinions of the participants around three themes: 1) reaction toward the study materials; 2) clarity and comprehensibility of the study materials; and, 3) participant consensus and divergent opinions regarding study materials. Each of the five topics discussed during the focus group and interviews was discussed until a consensus was reached about how to modify the study materials to take into account the views of the focus group participants. 
Table 1. Discussion Guide Topic Outline

\begin{tabular}{|c|c|}
\hline 1 & $\begin{array}{l}\text { Would you be more likely to participate in this study if you } \\
\text { were informed about it by a federal public health agency } \\
\text { (ATSDR), the state health department or by your neurologist? }\end{array}$ \\
\hline 2 & $\begin{array}{l}\text { Would you be more likely to participate if the questionnaire } \\
\text { was administered by phone or in person? }\end{array}$ \\
\hline 3 & $\begin{array}{l}\text { For the questionnaire, would you be willing to answer } \\
\text { questions regarding your: } \\
\text { a.Residential history } \\
\text { b.School history } \\
\text { c.Occupational history } \\
\text { d.Hobbies } \\
\text { e.Immunizations/vaccinations } \\
\text { f.Medical history } \\
\text { g.Symptoms related to your MS } \\
\text { h.Reproductive history } \\
\text { i.Medication } \\
\text { j.Family history }\end{array}$ \\
\hline 4 & $\begin{array}{l}\text { Would you be willing to have your blood drawn for } \\
\text { genotyping? }\end{array}$ \\
\hline 5 & $\begin{array}{l}\text { Would you be willing to go to a lab to have your blood drawn } \\
\text { or would you prefer to have someone come to your home? }\end{array}$ \\
\hline 6 & $\begin{array}{l}\text { How do you feel about not receiving the results of the } \\
\text { genotyping? }\end{array}$ \\
\hline 7 & $\begin{array}{l}\text { Would you be willing to have your blood sample stored for } \\
\text { future research on MS? }\end{array}$ \\
\hline
\end{tabular}

\section{Results}

\subsection{Demographics}

Nine individuals participated in the focus group and interviews. Six individuals participated in the face-to-face session and three were interviewed by telephone. The majority of participants $(n=8,89 \%)$ were female and all participants were white. Disease progression was variable among participants with some having an aggressive form and others an asymptomatic progression.

\subsection{Study Participation}

Overall, participants expressed interest in participating in the proposed case-control study. All agreed they would be more likely to participate if they were informed about the study by their neurologist and not a government public health agency. There was also consensus that study participation would not be affected if the questionnaire was administered by phone or in-person.

\subsection{Questionnaire}

All participants stated that they would be willing to answer questions regarding their past residential history, school history, and hobbies. Concerns were raised about people who had lived in numerous places over the course of their lifetime or who had worked in numerous occupations or job settings. The participants felt that it would take an inordinate amount of time to obtain the necessary information and that people might not be able to complete the entire questionnaire during one session. Questions were raised regarding specific portions of the questionnaire (i.e. symptoms of MS and types of medications taken) and what the responses would be used for. Participants felt strongly that the questionnaire should also include an open-ended question regarding what the individual felt caused their MS.

\subsection{Blood Sample}

Although all participants stated they would be willing to provide a blood sample for genotyping, they were not in agreement as to whether they would go to a lab to get their blood drawn. Individuals who did not have mobility problems were more willing to go to a lab to have their blood drawn, while those who had difficulty getting around stated that they would prefer to have someone come to their home to perform the blood draw.

\subsection{Genotyping}

All focus group participants were extremely upset when told that there were no plans to share individual results of the genotyping with study participants. Most of the focus group participants stated that they would not provide a blood sample if they would not receive their individual results.

\subsection{Other Issues}

Two other issues were identified during the focus group discussion:

1. All participants were concerned about protecting confidentiality including not identifying them as having MS and guaranteeing that their genotyping results would not be given to their insurance company or employer; and

2. Concern that some individuals with MS do not have the cognitive ability to answer the questions but could have legal guardians who could answer on their behalf.

\subsection{Changes Made}

Comments received from focus group participants were incorporated into the study protocol, study materials, and questionnaire. These included:

1. The introductory letter would be sent to potential participants from their neurologist and would not identify someone as having MS. The same letter would be sent to both cases and controls.

2. An information sheet was developed explaining the purpose of the study in a question and answer format.

3. Modification of the questionnaire included:

a) Specific time periods were added to the questions regarding residence, occupation etc. to limit the amount of responses;

b) an open-ended question of what a participant thought caused their MS was added; 
c) the list with symptoms associated with MS was revised;

d) the medication section of the questionnaire was deleted.

4. Study participants would have a choice where to have their blood drawn (i.e. at a lab or at home).

5. The introductory materials were revised to state more strongly that this study was research and the results of the genetic testing would not be provided to participants except in summary form since they have no clinical significance. The protocol was modified to state that if the genotyping information became clinically relevant then the results would be provided to study participants.

6. Confidentiality would be assured by including the study identification number only on the blood samples and only the Principal Investigator at ATSDR would have access to both questionnaire and genetic information.

7. Study participation would be limited to those with the cognitive ability to respond and answer questions.

8. A worksheet was developed for participants to complete before the questionnaire so that study participants would have time to obtain the necessary information.

9. Times for the phone interview would be flexible (i.e. day, night, weekends) and if the participant was unable to complete the questionnaire at one sitting the interview would be continued at a different time.

10. If a participant was unable to complete the questionnaire over the phone, a hard copy would be sent to them to complete.

11. The consent form was modified to include a separate section about storing the remaining blood sample for future studies of MS.

\section{Conclusion}

Conducting formative research can provide significant return to the study investigator and to study participants. In addition to insights provided from these activities to investigators, participants can pilot study materials allowing problems to be identified and corrected before study recruitment begins. It also allows investigators the opportunity to modify the protocol and materials to effectively meet the needs of potential study participants and promote participation. Utilizing individuals who are similar to the targeted participants in the proposed study is indispensable in making sure that the study materials are understandable and appropriate [9]. The result was that the majority of the participants in the case-control study that was completed in 2012 provided a blood sample for genotyping (91\%) and consented to have their blood sample stored for future MS research (97.5\%) [12].Feedback from our focus group participants indicated that the participatory process also provided a welcome opportunity for them to contribute to efforts to better understand the disease with the ultimate hope of benefitting others with MS and their families.

\section{Discussion}

Recruiting and retaining participants in epidemiologic studies is always a fundamental concern and vital to any study's success. However, conducting formative research before the onset of a study to obtain feedback on materials is not ordinarily done. Before embarking on a case-control study of MS, we were worried about non-participation because study participants would be asked to provide a blood specimen for genotyping. To address this concern, we held discussions with individuals with MS and asked them to help to develop effective and sensitive study materials. Taking the time to conduct formative research can be beneficial even though adding another step in the study development takes time and resources, both of which are typically in limited supply.

The main strengths of the focus group discussions were that the individuals who participated represented the case population that would be targeted in the proposed study and that they were very open and forthcoming with their opinions. This openness could be attributed, at least in part, to the participants having the disease being investigated and being motivated to help improve the study. In addition, the facilitators were individuals they knew or had been in contact with and so rapport had already been established.

Some of the limitations of our approach were that we conducted a very simplistic focus group discussion with individuals who had MS but who were more positive toward the conduct of research activities. We also only conducted one focus group discussion which had a small sample size of homogeneous individuals. These limitations may have had an impact on the range of viewpoints that were collected and therefore on the changes made to the study materials.

Even with these limitations, the formative research provided us with information that was indispensable to the proposed case-control study. Feedback from participants indicated that the participatory process provided a welcome opportunity for them to contribute to efforts to better understand their disease with the ultimate hope of benefitting others with MS. There is always room for improvement so we recommend the following:

1. Budget time and resources to conduct multiple representative focus groups.

2. Be very clear about what you want to accomplish with the focus group discussion. Go through the study materials and identify areas that you want focus groups participants to comment on.

3. The questions posed to the focus group participants should not convey the bias of the investigators.

4. Make an agenda for the meeting and share it with participants so they know what is expected of them. Assure them that discussion is welcome and encouraged. Clearly defining areas you want to 
concentrate on will help make the analytic process more efficient.

5. Make sure all study personnel are available during the meeting.

6. Have participants review all study materials.

7. Make sure that the confidentiality of participants is protected at all times.

8. During the focus group discussion make sure everyone has the opportunity to speak - just because someone hasn't said something doesn't mean they agree with what is being said.

\section{Acknowledgements}

The authors would like to thank the individuals who participated in the focus group discussion.

\section{Disclaimer}

The findings and conclusions in this article are those of the authors and so not necessarily represent the official position of the Centers for Disease Control and Prevention.

\section{REFERENCES}

[1] Catz DS, Green NS, Tobin JN, et al.Attitudes about genetics in underserved, culturally diverse populations. Community Genetics 2005;8:161-172.

[2] Burke W, Atkins D, Gwinn M, et al. Genetic Test Evaluation: Information Needs of Clinicians, Policy Makers, and the Public. Am J Epidemiol 2002;156:311-318.
[3] Galea S,Tracy M. Participation Rates in Epidemiologic Studies. Ann Epidemio 2007;17:643-653.

[4] Morton LM. Encouraging participation in medical research: what strategieswork? J ClinEpidemiol 2008;61: 969-970.

[5] O’Daniel J,Haga SB.Public perspectives on returning genetics and genomics research results. Public Health Genomics 2011;14:346-355.

[6] Gittelsohn J, Steckler A, Johnson CC, et al. Formative Research in School and Community-Based Health Programs and Studies: "State of the Art" and the TAAG Approach. Health EducBehav2006;33(1):25-39.

[7] Bates BR, Lynch JA, Bevan JL, Condit CM. Warranted concerns, warranted outlooks: a focus group study of public understandings of genetic research. SocSci Med 2005;60:331-334.

[8] Lobdell DT, Gilboa S, Mendola P, Hesse BW. Use of focus groups for the environmental health researcher. J Env Health 2005;67:36-42.

[9] Streicher SA, Sanderson SC, Jabs EW, et al. Reasons for participating and genetic information needs among racially and ethnically diverse biobank participants: a focus group study. Community Genetics 2011;2:153-163.

[10] Lasset C, Charavel M, Bonadona V. Focus group approach for developing written patient information in oncogenetics. Genotyping 2007;11:193-197

[11] Henry JP, Williamson DM, Schiffer R, Wagner L, Shire J, Garabedian M. Investigation of a cluster of multiple sclerosis in two elementary school cohorts. J Environ Health 2007;69: 34-40.

[12] Williamson DM, Marrie RA, Ashley-Koch A, Schiffer R, Trottier J, Wagner L. Design, methodological issues and participation in a multiple sclerosis case-control study. ActaNeurolScand 2012;126:197-204. 